accepted by ApJ Letters

\title{
Relics of Double Radio Sources
}

\author{
K. S. Dwarakanath \& Ruta Kale \\ Raman Research Institute, Bangalore 560 080, India \\ dwaraka@rri.res.in, ruta@rri.res.in
}

\begin{abstract}
We have formed a new sample which consists of extended extragalactic radio sources without obvious active galactic nuclei (AGN) in them. Most of these sources appear to be dead double radio sources. These sources with steep spectra $\left(\alpha<-1.8 ; \mathrm{S} \propto \nu^{\alpha}\right)$ were identified using the 74 (VLSS) and the $1400 \mathrm{MHz}$ (NVSS) surveys and further imaged using the Very Large Array (VLA) and the Giant Meterwave RadioTelescope (GMRT). The radio morphologies of these sources are rather unusual in the sense that no obvious cores and jets are detected in these sources, but, two extended lobes are detected in most. The mean redshift of 4 of the 10 sources reported here is $\sim 0.2$. At a redshift of 0.2 , the linear extents of the sources in the current sample are $\sim 250 \mathrm{kpc}$ with their spectral luminosities at $1.4 \mathrm{GHz}$ in the range $2-25 \times 10^{23} \mathrm{~W} \mathrm{~Hz}^{-1}$. The steep spectra of these sources is a result of the cessation of AGN activities in them about 15-100 million years ago. Before the cessation of AGN activity, the radio luminosities of these galaxies were $\sim 1000$ times brighter than their current luminosities and would have been comparable to those of the brightest active radio galaxies detected in the local universe $\left(\mathrm{L}_{1.4} \sim 10^{27} \mathrm{~W} \mathrm{~Hz}^{-1}\right)$. The dead radio galaxies reported here represent the 'tip of the iceberg' and quantifying the abundance of such a population has important implications to the life cycle of the AGN.
\end{abstract}

Subject headings: galaxies: active — galaxies: halos — galaxies: high-redshift — radiation mechanisms: non-thermal — radio continuum: galaxies

\section{INTRODUCTION}

Sources with steep spectra $\left(\alpha<-1.5 ; \mathrm{S} \propto \nu^{\alpha}\right)$ at low radio frequencies $(\nu<\sim 1 \mathrm{GHz})$ are interesting to study from many points of view. Such sources could be (a) pulsars (millisecond, 
or, otherwise), (b) haloes in galaxy clusters, (c) relics of double radio sources, or, (d) high redshift radio galaxies. Although sources of this kind are known, more in some category than in the other, they are rather rare. Multi frequency studies of these objects are even rarer. In this background, it is interesting to follow up steep-spectrum sources identified from sensitive low-frequency surveys.

Several studies of steep spectrum sources extracted from various radio surveys have been carried out. Based on the radio surveys at 38 and $178 \mathrm{MHz}$, Baldwin and Scott discussed sources with spectra steeper than -1.2 and concluded that about half of these sources are associated with clusters of galaxies (Baldwin \& Scott, 1973). Komissarov and Gubanov (1994) modeled the evolution of the synchrotron spectrum of a number of steep-spectrum sources $(\alpha<-2.0)$ found in the centers of rich clusters. The spectra of radio relics in galaxy clusters were modeled by Slee et al (2001) and Kaiser and Cotter (2002). Gopal-Krishna et al (2005) extracted a sample of 52 sources from the Ooty lunar occultation survey, Molonglo $408 \mathrm{MHz}$ surveys MC1, MC2 and MIT-Greenbank survey. The spectral indices of sources in this sample in the frequency range $300 \mathrm{MHz}-5 \mathrm{GHz}$ are steeper than -1.1 but flatter than -1.5 . This study detected many radio sources in the redshift range $0.4-2.6$, some of them in galaxy clusters. There appear to be some radio sources in this sample which are at redshifts even beyond 2.6. Klamer et al (2006) have studied a sample of 76 steep spectrum ( $\alpha$ in the range $-1--1.6$ ) sources extracted from the SUMSS and the NVSS surveys to find high $\mathrm{z}$ radio galaxies and advance a plausible cause behind the $\mathrm{z}-\alpha$ correlation. Cruz et al. (2006) studied a sample of 68 sources extracted from the $6 \mathrm{C}(151 \mathrm{MHz})$ survey. The spectral indices (between 151 and $1400 \mathrm{MHz}$ ) of these sources are in the range -1 - -1.4 and these sources are confined to $\sim 0.4$ sr of the sky. The redshifts of these sources are in the range 0.2 - 3.3. Parma et al (2007) studied a sample of steep-spectrum $(\alpha<-1.3)$ sources selected by comparing the NVSS and the WENSS catalogs and found six dying sources and three restarted sources.

Recently, results from the VLA $74 \mathrm{MHz}$-survey have become available. This survey has a resolution of $\sim 80^{\prime \prime}$ with an average $\mathrm{rms}$ of $\sim 0.1 \mathrm{Jy} /$ beam (Cohen et al 2006). In the Data Release I the area covered is $\sim 6 \mathrm{sr}$. This survey is the most sensitive survey to date in this frequency range. This survey data can be effectively used to identify the steep-spectrum sources. On similar lines of motivation, a limited area of the sky $(\sim 0.05 \mathrm{sr})$ was analyzed by Cohen at al (2004) and further followed by imaging in the near-infrared by Jarvis et al (2004). The radio sources in their list had spectral indices (between 74 and $1400 \mathrm{MHz}$ ) in the range $-1.2--1.8$. These studies have yielded potentially interesting candidates for high redshift radio galaxies, relics of double radio sources, and cluster halo sources. 


\section{STEEP-SPECTRUM SOURCES}

In order to select steep-spectrum sources, the Data Release I of the VLA 74-MHz survey (VLSS) and the $1.4 \mathrm{GHz}$ NRAO VLA Sky Survey (NVSS) were used. The resolutions of the VLSS and of the NVSS surveys are $\sim 80^{\prime \prime}$ and $45^{\prime \prime}$ respectively. The detection limits of the VLSS and the NVSS surveys are $\sim 0.5 \mathrm{Jy} /$ beam and $2 \mathrm{mJy} /$ beam respectively corresponding to 5 times the RMS in the respective images. The source positions in the VLSS survey $(\sim$ 32000 sources) were compared with those of the NVSS sources $(\sim 1.8$ million sources $)$. More than $\sim 80 \%$ of the sources in the VLSS had a counterpart in the NVSS within $\sim 15^{\prime \prime}$ - well within the expected value based on positional uncertainties in the two surveys. The spectral indices (flux density $\propto \nu^{\alpha}$ ) of all these sources were estimated between 1400 and $74 \mathrm{MHz}$. The distribution of spectral indices mimics a Gaussian distribution with a mean spectral index of -0.79 and an rms of 0.2 . These values are consistent with those obtained in earlier higher frequency surveys.

For the purposes of this study, 'steep-spectrum sources' are those which have spectral indices smaller than, or, equal to -1.8 (mean-5 $\sigma$ ). These are continuum sources with the steepest spectra that have been studied yet. There were 38 such sources in the VLSS. The NASA Extragalactic Database (NED) and the SIMBAD were searched for any other information about these sources. Of the 38, only 12 of them had non-stellar sources within an arc minute of their positions. In addition, there are 3 more sources which do not have an optical counterpart, but are considered 'extended' based on the NVSS data. Given the complementary information they have, these 15 sources were further followed up with imaging at low-frequencies.

\section{OBSERVATIONS AND DATA ANALYSIS}

Of these 15 sources, two sources $0741+7414$ (in $\mathrm{ZwCl} 0735.7+7421$ ) and 0041-0923 (in Abell 85) have already been imaged at 330 and $1420 \mathrm{MHz}$ with the VLA (Cohen et al, 2005; Slee et al 2001; Young 2004) with interesting results regarding the nature of these steep spectrum sources. The remaining 13 sources were observed at $330 \mathrm{MHz}$ with the VLA in the A configuration $\left(\sim 10^{\prime \prime}\right.$ resolution) during June - Sep, 2007. The observations were carried out in the $4 \mathrm{IF}$ mode with a bandwidth of $6.25 \mathrm{MHz}$ and 16 channels. The two frequencies were centered at 321.5 and $328.5 \mathrm{MHz}$ respectively. In each observing session, 3 to 4 sources were cycled through for a total of 6 to 8 hours giving an integration of time $\sim 2$ hours on each source, but with a better visibility coverage.

Of the 13 sources observed with the VLA, 4 sources had poor quality data from the 
VLA to EVLA transition-related problems. They were discarded. The remaining 9 sources were observed with the GMRT. The observations with the GMRT were carried out during 5 - 15 May, 2008. Observations were carried out at a center frequency of $1287 \mathrm{MHz}$ with a 16 $\mathrm{MHz}$ bandwidth and 128 channels. Two sources were cycled through in an observing session with a total duration of $\sim 10$ hours, giving an integration time on each source $\sim 5$ hour.

Both the VLA and the GMRT data were analyzed using the Astronomical Image Processing System. The multichannel nature of the continuum data was effective in identifying and excising the radio frequency interference (RFI). About $10-30 \%$ of the data was lost to RFI in the VLA and in the GMRT data.

\section{RESULTS}

All the 9 sources except one were detected at both the frequencies. The undetected source was a result of confusion from a brighter source in the lower-resolution VLSS and NVSS surveys. Images of the relevant portions of the 6 (out of 8 ; to conform to the Letters length ) fields at 328 and $1287 \mathrm{MHz}$, convolved to the common resolution, are displayed in Figs. 1-3. The integrated flux densities of the steep-spectrum sources estimated from these images are given in Table 1. This table has 10 entries since one of the fields (0128) has three sources with different spectral indices. In the case of rest of the sources, the spectral indices of the two lobes were estimated separately and were found to be in agreement with each other and with the respective integrated spectral indices within the errors (Table 1). Images from the NVSS and VLSS were used to estimate the corresponding flux densities at 1400 and $74 \mathrm{MHz}$ respectively. The flux density values at $74 \mathrm{MHz}$ are given in Table 1 . Where available, the flux densities of the sources at $326 \mathrm{MHz}$ were also extracted from the WSRT catalog. The mean differences between the VLA (at $328 \mathrm{MHz}$ ) and the WSRT (at $326 \mathrm{MHz}$ ) flux densities and that between the GMRT (at $1287 \mathrm{MHz}$ ) and the NVSS (at $1400 \mathrm{MHz}$ ) flux densities are $14 \%$ and $28 \%$ respectively. The spectral indices estimated based on the flux densities from the current observations and from the $74 \mathrm{MHz}$ survey are given in Table 1. The spectra of all the sources in Figs. 1-3 are displayed in Fig. 4.

\section{DISCUSSION}

A striking feature of the morphologies of the steep-spectrum sources is their doublelobed nature in most cases. The morphologies of the steep-spectrum sources observed here are similar to those of radio galaxies rather than that of halos and relics observed in galaxy 
clusters. Furthermore, there are no clear indications of cores and jets in any of these sources (with the exception of 0128B) even in the highest resolution $\left(\sim 4^{\prime \prime}\right)$ GMRT images at 1287 $\mathrm{MHz}$. The morphologies, the absence of cores and jets and the steep spectra of these sources imply that they are most likely fossil radio sources. There is no evidence that any of these sources reside in galaxy clusters except perhaps the sources 1152 and 2216. In contrast to this, both the steep-spectrum sources from the VLA $74 \mathrm{MHz}$ survey imaged in recent times turned out to be in galaxy clusters (Slee et al 2001; Cohen et al 2005).

Four of the ten sources are at a redshift of $\sim 0.2$ (see notes to Table 1 ). For the purposes of this discussion we will assume the current sample of steep-spectrum sources to be at a redshift of 0.2 . This is not a serious drawback since the conclusions we will draw on the nature of these sources is not sensitive to this assumption. The implied range of radio luminosities $\left(\mathrm{H}_{o}=75 \mathrm{~km} \mathrm{~s}^{-1} \mathrm{Mpc}^{-1}\right)$ at $1400 \mathrm{MHz}$ is $2-25 \times 10^{23} \mathrm{~W} \mathrm{~Hz}^{-1}$, close to the value where the distribution of local AGNs peak (Condon 1989, Sadler et al 2002). The linear extents of the steep-spectrum sources are in the range $100-400 \mathrm{kpc}$. The equipartition magnetic fields in these systems are in the range $2-10 \mu \mathrm{G}$.

Several previous studies of spectral evolution and modeling exist to account for steep spectra (Komissarov \& Gubanov 1994, Goldshmidt \& Rephaeli 1994, Slee et al 2001, Ensslin \& Gopal-Krishna 2001). The basic synchrotron theory is described in Kardashev (1962) and Pacholczyk (1970). Spectral steepening in extragalactic radio sources is due to differential energy loss of the relativistic electrons as a function of their energy (E). The energy losses relevant for the sources of interest are synchrotron, inverse-Compton and adiabatic losses. Synchrotron losses are proportional to $\mathrm{B}^{2} \mathrm{E}^{2}$ while the inverse Compton losses are proportional to $\mathrm{B}_{i c}^{2} \mathrm{E}^{2}$, where, $\mathrm{B}$ is the magnetic field strength in the source and $\mathrm{B}_{i c}(=$ $\left.3.25(1+z)^{2} \mu \mathrm{G}\right)$ is the magnetic field strength with energy density equal to that of the cosmic microwave background. The adiabatic losses are connected to the change in the volume of the source. Ensslin and Gopal-Krishna (2001) set up a formalism in which they start with the equation relating the change in the energy of the relativistic electrons with the losses mentioned above. They further assume sufficient pitch angle scattering to keep the electron pitch angle distribution isotropic (JP model; Jaffe \& Perola 1973). The solution to this equation leads to the expression for the synchrotron luminosity at any given frequency, $\mathrm{L}_{\nu i}=c_{3} B_{i} V_{i} \int_{p m i n i}^{p \operatorname{maxi}} d p f_{i}(p) F\left(\nu / \nu_{i}(p)\right)$ (eq. 19 in their paper). In this equation, $c_{3}$ is a constant and $F\left(\nu / \nu_{i}(p)\right)$ is the dimensionless spectral emissivity of a mono-energetic isotropic electron distribution in isotropically oriented magnetic fields. The symbols $B_{i}$ and $V_{i}$ are the magnetic field and the volume of the source respectively in the i'th phase. In the current study, the two phases considered are the injection and the expansion phases. Starting from an initial power law, the resulting electron spectrum (due to the losses mentioned earlier) in 
the i'th phase is given by $f_{i}(p)$. This equation does not have an analytical solution and was evaluated numerically in the current study. A more detailed discussion of this formalism is beyond the scope of this Letter (see Ensslin \& Gopal-Krishna (2001) for details).

The above formalism was used to estimate the model spectra that best-fit the observed data points. The input parameters to the computation of the model spectra are (a) the current volume of the source, (b) the source magnetic field and (c) the initial index $\gamma$ in the energy distribution of the relativistic electrons $\left(\mathrm{N}=\mathrm{N}_{o} \mathrm{E}^{-\gamma}\right)$. The spectra were obtained at any given time, $\mathrm{t}=\mathrm{t}_{C I}+\mathrm{t}_{R E}$, where $\mathrm{t}_{C I}$ is the duration of the continuous injection phase and $t_{R E}$ is the duration of the relic phase during which the injection of relativistic electrons is switched off (see Slee et al 2001). It is possible to estimate the parameters $\left(\right.$ viz. $\mathrm{t}_{C I}, \mathrm{t}_{R E}$, and B) for each source that best-fit the respective spectra.

The current volume of the source was estimated for a linear size of $250 \mathrm{kpc}$. This linear size corresponds to an angular size of $\sim 1^{\prime}$ (see Fig. 1-3) at a redshift of $0.2\left(\mathrm{H}_{o}=75 \mathrm{~km}\right.$ $\left.\mathrm{s}^{-1} \mathrm{Mpc}^{-1}\right)$. A value of $\gamma=2.5$ was assumed corresponding to an $\alpha=-0.75$, where the flux density is $\propto \nu^{\alpha}$. For different sources, a range of $\mathrm{t}_{C I}$ 's, $\mathrm{t}_{R E}$ 's and B's were considered and the model spectra obtained. The model spectra were then compared with the observed data. The model spectrum with a single value of B has an exponential high frequency cut-off and is unable to fit the observed data. This is a well-known problem as was noted by Komissarov \& Gubanov (1994) and Slee et al (2001). However, if the spectra for a range of field strengths is obtained and combined, the sharp JP cutoff is smoothed out and the integrated spectrum fits the data well. Such a model is considered more physical due to the different strengths of magnetic fields believed to exist in any of these systems (Slee et al 2001). The solid lines in Fig. 4 indicate the best-fit model spectra to the corresponding data points. The model spectra in each case were anchored to the flux density at $74 \mathrm{MHz}$ to fix the y-normalization. The dotted and dashed lines in Fig. 4 indicate the model spectra when $\mathrm{t}_{R E}$ was changed by $\pm 30 \%$ respectively with respect to the best-fit values quoted in each panel. It is evident from these plots that a $\pm 30 \%$ change from the best-fit value of $t_{R E}$ produces model spectra which are in disagreement with the observed data points and predicts values beyond the $3 \sigma$ errors of the measurements. So, the errors in the estimates of $t_{R E}$ 's are not more than $\pm 30 \%$. Similar considerations indicate that the errors in the estimates of $t_{C I}$ 's are not more than $\pm 50 \%$. Although there are only three data points, the shape of the spectrum is well-constrained since the data points are well-separated in frequency. Since the shape of the spectrum is a sensitive function of $\mathrm{t}_{C I}, \mathrm{t}_{R E}$ and $\mathrm{B}$ it has been possible to estimate these parameters. The ranges of parameters that account for the observed spectra of the current sample of sources are the following : $10<\mathrm{t}_{C I}<400 \mathrm{My}, 15<\mathrm{t}_{R E}<90 \mathrm{My}$ and $2<\mathrm{B}<10$ $\mu \mathrm{G}$. The mean value of the ratio $t_{R E} / t_{C I}$ for the current sample of sources (Table 1 ) is 1.3 . This mean value of $\mathrm{t}_{R E} / \mathrm{t}_{C I}$ implies that most of the sources discussed here are at a stage 
when the central engine has been off for as long as it was on. During the computation of model spectra, it is also possible to keep track of the relative decrease in the radio luminosity as a function of time. This ratio varies in the range $10^{2}$ to $10^{4}$ depending on the value of $\mathrm{t}_{R E} / \mathrm{t}_{C I}$ for different sources. Since the current radio luminosities of these sources are $\sim 10^{24}$ $\mathrm{W} \mathrm{Hz}{ }^{-1}$ at $1.4 \mathrm{GHz}$, the peak radio luminosities of these sources when the AGN was active would have been $\sim 10^{27} \mathrm{~W} \mathrm{~Hz}^{-1}$, brighter than the brightest of the currently known radio galaxies. While the current sample represents sources when the relic phase is comparable to the active phase, the Parma et al (2007) sample represents radio galaxies when the relic phase is an order of magnitude smaller than the active phase.

The detection limits of the surveys at 1400 and $74 \mathrm{MHz}$ are 2 and $500 \mathrm{mJy} /$ beam respectively. The sensitivities of these two surveys are comparable for sources with a spectral index of -1.8. Hence, such steep-spectrum sources detected at $1400 \mathrm{MHz}$ would also be detected at $74 \mathrm{MHz}$. The peak luminosities of the sources discussed here is $\mathrm{L}_{1.4} \sim 10^{27}$ $\mathrm{W} \mathrm{Hz}{ }^{-1}$, when they were active. Most of these sources are close to the detection limits of the two surveys used to identify them. However, the luminosity function of currently active AGNs indicates that their number density is about 100 times higher at $\mathrm{L}_{1.4} \sim 10^{24} \mathrm{~W} \mathrm{~Hz}^{-1}$ (Sadler et al 2002) than at $\mathrm{L}_{1.4} \sim 10^{27} \mathrm{~W} \mathrm{~Hz}^{-1}$. At the corresponding ages of the sources reported here, majority of the currently active sources (with $\mathrm{L}_{1.4} \sim 10^{24} \mathrm{~W} \mathrm{~Hz}^{-1}$ ) will remain undetected at the current sensitivity limits of low-frequency surveys. Hence, the dead radio sources discussed here represent just the 'tip of the iceberg' with a large population of such sources to be unearthed from more sensitive low frequency surveys. Such surveys ought to detect a large number of AGNs at different stages of their activities (and hence different spectral indices) and lead to an understanding of their workings and evolution.

We thank Biman Nath and Ravi Subrahmanyan for critical comments and discussions. The National Radio Astronomy Observatory is a facility of the National Science Foundation operated under cooperative agreement by Associated Universities, Inc. GMRT is run by the National Centre for Radio Astrophysics of the Tata Institute of Fundamental Research. 


\section{REFERENCES}

Baldwin, J. E., Scott, P. F. 1973, MNRAS, 165, 259.

van Breugel, W., De Breuck, C., Stanford, S. A., Stern, D., Rottgering, H., Miley, G. 1999, ApJ, 518, L61.

Cohen, A. S., Rottgering, H. J. A., Jarvis, M. J., Kassim, N. E., Lazio, T. J. W. 2004, ApJSS, 150, 417.

Cohen, A. S., Clarke, T. E., Feretti, L., Kassim, N. E. 2005, ApJL, 620, 5.

Cohen, A. S., Lane, W. M., Kassim, N. E., Lazio, T. J. W., Cotton, W. D., Perley, R. A., Condon, J. J., Erickson, W. C. 2006, AN, 327, 262.

Condon, J. J. 1989, ApJ, 338, 13.

Cruz, M. J., Jarvis, M. J., Blundell, K. M., Rawlings, S., Croft, S., Klockner, H., McLure, R. J., Simpson, C., Targett, T. A., Willott, C. J. 2006, MNRAS, 373, 1531.

Ensslin, T. A., Gopal-Krishna 2001, A\&A, 366, 26.

Goldshmidt, O., Rephaeli, Y. 1994, ApJ, 431, 586.

Gopal-Krishna, Ledoux, C., Melnick, J., Giraud, E., Kulkarni, V., Altieri, B. 2005, A\&A, $436,457$.

Jaffe, W. J., \& Perola, G. C. 1973, A\&A, 26, 423

Jarvis, M. J., Cruz, M. J., Cohen, A. S., Rottgering, H. J. A., Kassim, N. E. 2004, MNRAS, $355,20$.

Kaiser, C. R., Cotter, G. 2002, MNRAS, 336, 649.

Kardashev, N. S. 1962, SvA, 6, 317.

Klamer, I. J., Ekers, R. D., Bryant, J. J., Hunstead, R. W., Sadler, E. M., De Breuck, C. 2006, MNRAS, 371, 852.

Komissarov, S. S., Gubanov, A. G. 1994, A\&A, 285, 27.

Pacholczyk, A. G. 1970, Radio Astrophysics (San Francisco: Freeman)

Parma, P., Murgia, M., de Ruiter, H. R., Fanti, R., Mack, K.-H., Govoni, F. 2007, A\&A, $470,875$. 
Sadler, E. M. et al 2002, MNRAS, 329, 227.

Slee, O. B., Roy, A. L., Murgia, M., Andernach, H., Ehle, M. 2001, AJ, 122, 1172.

Young, A. 2004, PhD Thesis, University of Minnesota.

This preprint was prepared with the AAS LATEX macros v5.2. 

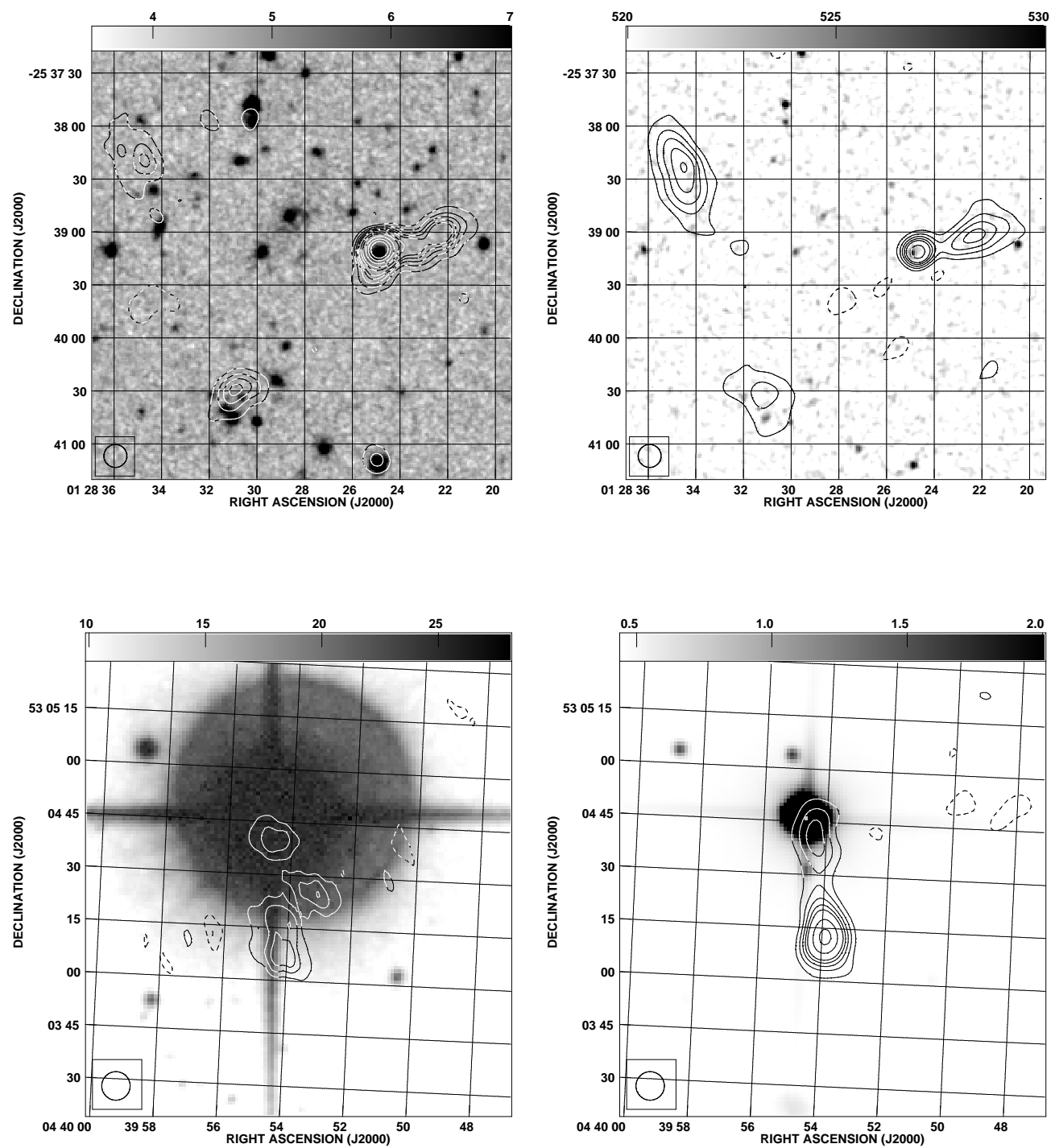

Fig. 1.- top (0128) : GMRT (left, $1287 \mathrm{MHz}$ ) and VLA (right, $328 \mathrm{MHz}$ ) radio images (contours) overlaid on optical and $2 \mu$ All Sky Survey images (in grey) respectively. The synthesized beam is $13^{\prime \prime} \mathrm{X} 13^{\prime \prime}$ and the rms values are 0.14 (left) and 3.5 (right) mJy/beam. The contours are at $-0.5,0.5,1,1.5,2,2.5$ and 3.75 (left) and at -8, 8, 16, 24, 32, 40, 60, 80, 120, 160 and 200 (right) mJy/beam. The source to the NE is $0128 \mathrm{~A}$, to the NW is $0128 \mathrm{~B}$ and to the south is $0128 \mathrm{C}$. bottom (0439): The synthesized beam is $8^{\prime \prime} \mathrm{X} 8^{\prime \prime}$. The rms values are 0.14 (left) and 1.6 (right) mJy/beam. The contours are at -0.5, 0.5, 1 and 1.5 (left) and at $-4,4,8,12,16,20,30$ and 40 (right) mJy/beam. 

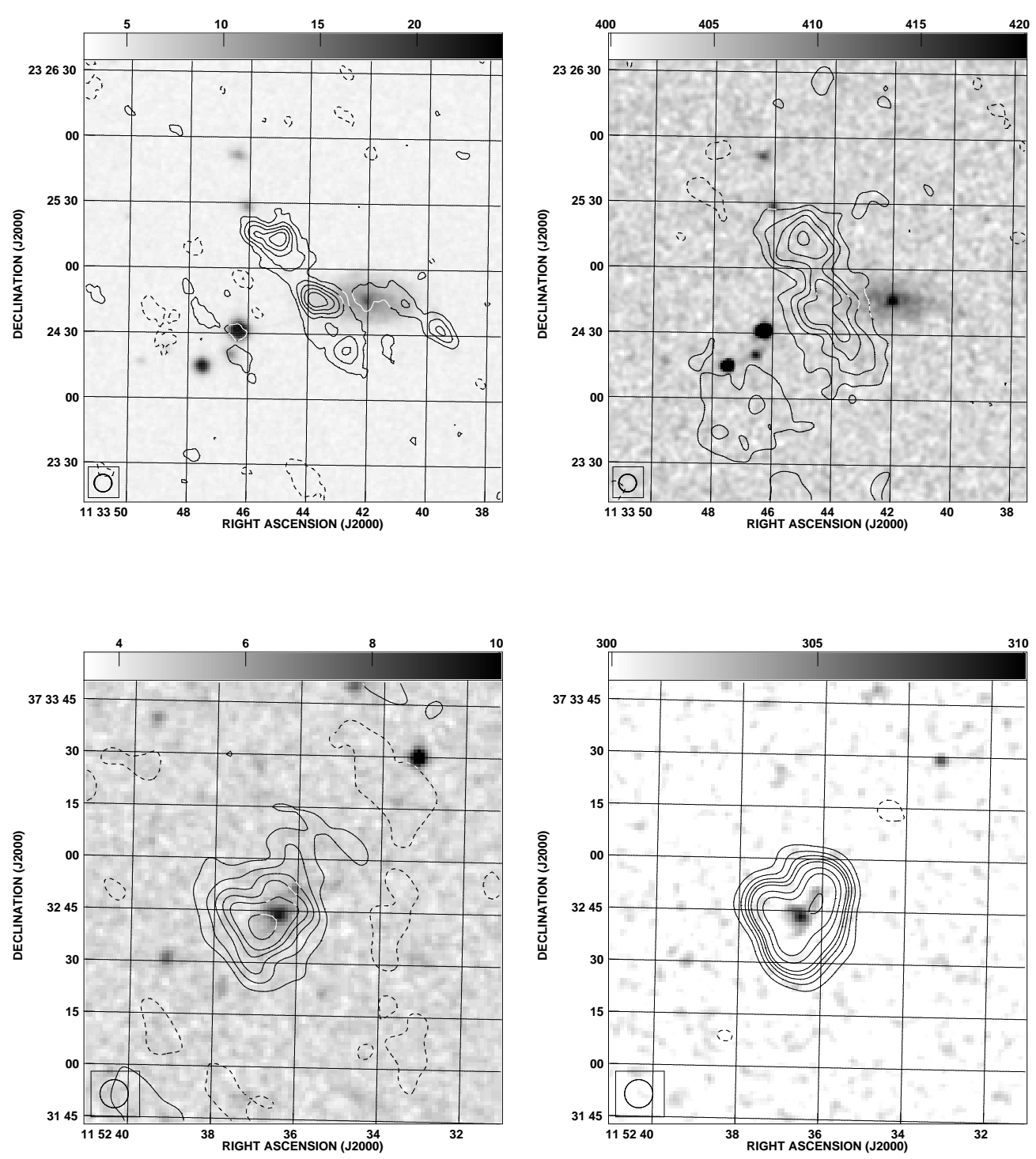

Fig. 2.- top (1133): The synthesized beam is $8^{\prime \prime} \mathrm{X} 8^{\prime \prime}$. The rms values are 0.1 (left) and 0.95 (right) $\mathrm{mJy} /$ beam. The contours are at $-0.2,0.2,0.4,0.6,0.8$ and 1 (left) and at $-2,2$, 4, 6, 8 and 10 (right) mJy/beam. bottom (1152): The synthesized beam is $8^{\prime \prime} \mathrm{X} 8^{\prime \prime}$. The rms values are 0.3 (left) and 1.3 (right) mJy/beam. The contours are at $-0.6,0.6,1.2,1.8$, 2.4, 3 and 4.5 (left) and at -4, 4, 8, 12, 16, 20, 30, 40 and 60 (right) mJy/beam. 

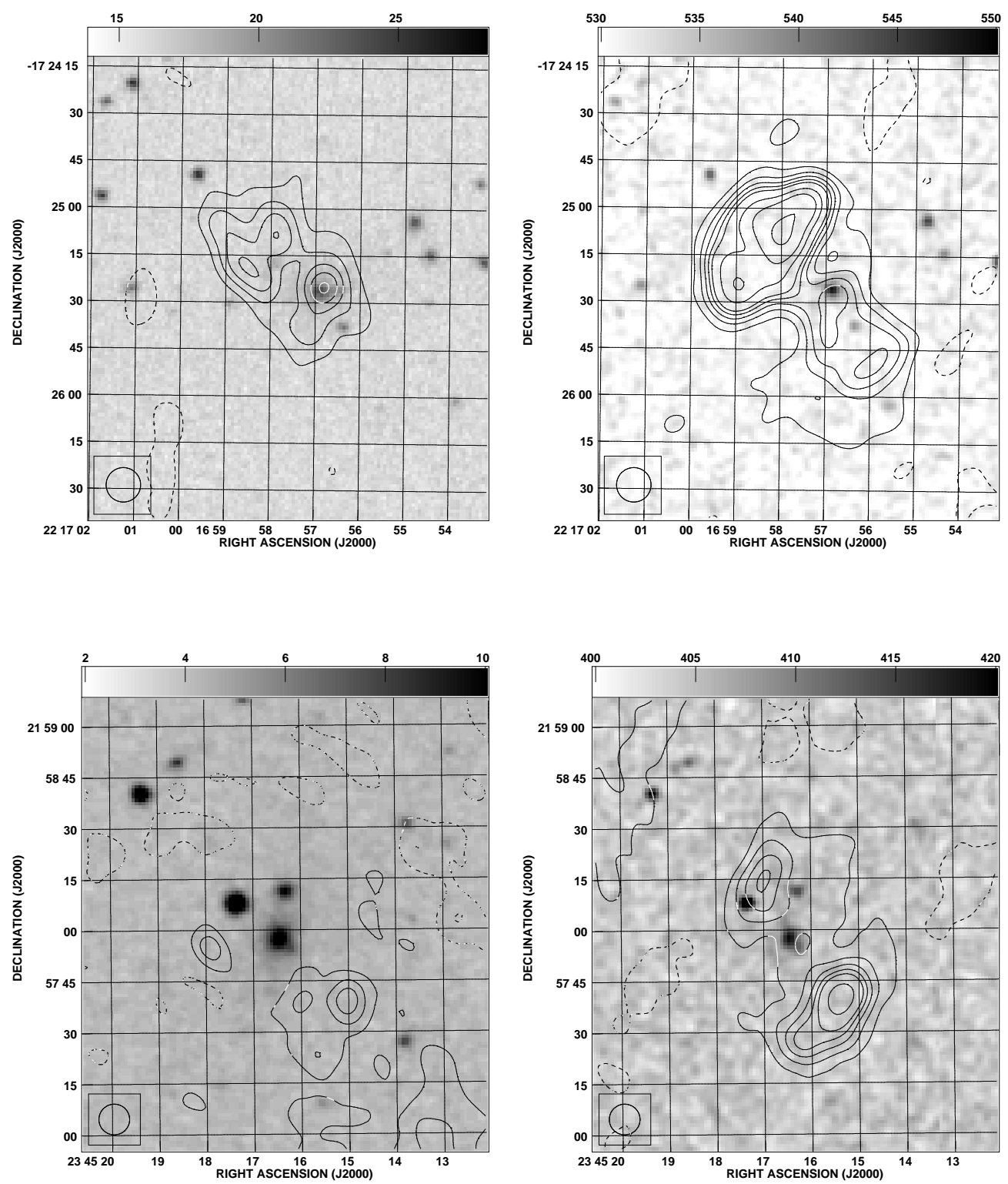

Fig. 3.- top (2216): The synthesized beam is $11^{\prime \prime} \mathrm{X} 11^{\prime \prime}$. The rms values are 0.18 (left) and 2.7 (right) mJy/beam. The contours are at -0.4, 0.4, 0.8, 1.2, 1.6 and 2.0 (left) and at -4, 4, 8, 12, 16, 20, 30, 40 and 60 (right) mJy/beam. bottom (2345): The synthesized beam is $9^{\prime \prime} \mathrm{X} 9^{\prime \prime}$. The rms values are 0.44 (left) and 3.0 (right) $\mathrm{mJy} /$ beam. The contours are at $-0.7,0.7,1.4$ and 2.1 (left) and at -5.6, 5.6, 11.2, 16.8, 22.4, 28 and 42 (right) mJy/beam. 


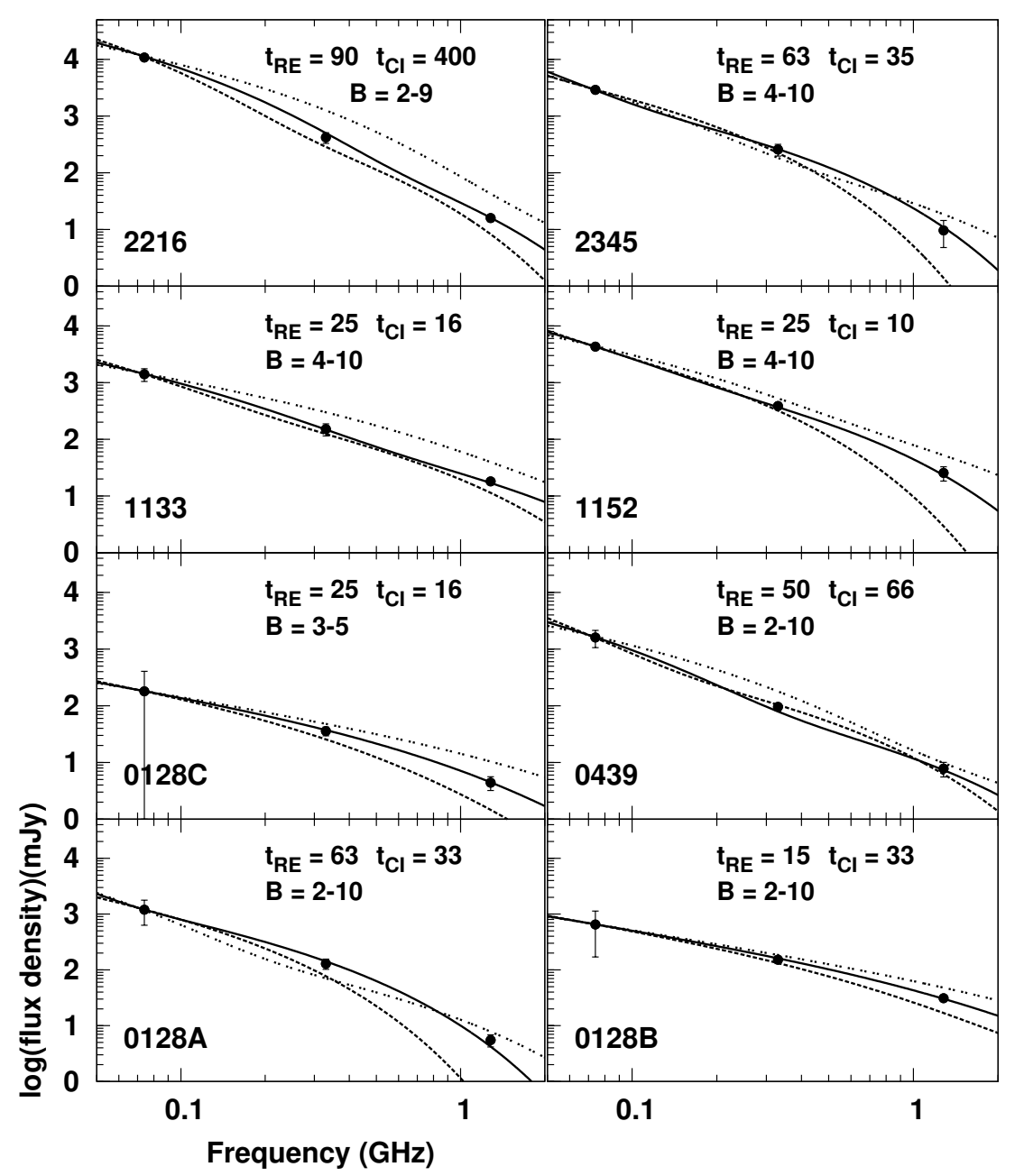

Fig. 4.- Spectra of all the sources in Figs. 1-3. The solid points are observed data along with $3 \sigma$ error bars. The solid line is the best-fit model spectrum. The best-fit parameters are indicated in each panel. The values of $\mathrm{t}_{R E}$ and $\mathrm{t}_{C I}$ are in million years and of $\mathrm{B}$ are in $\mu \mathrm{G}$. The dotted and dashed lines are model spectra when $\mathrm{t}_{R E}$ is changed by $\pm 30 \%$ respectively with respect to the best-fit value. The $3 \sigma$ errors are on the $t_{C I}$ values are $\pm 50 \%$. Note that the model spectra for each source are anchored to the respective data value at $74 \mathrm{MHz}$ to fix the y-normalization. 
Table 1. GMRT \& VLA Observations

\begin{tabular}{cccccccccc}
\hline \hline Source & $\begin{array}{c}\text { R.A. (J2000) } \\
\text { hh mm ss }\end{array}$ & $\begin{array}{c}\text { Dec. (J2000) } \\
\text { o }\end{array}$ & $\begin{array}{c}\text { S74 } \\
\text { (VLSS) }\end{array}$ & $\begin{array}{c}\text { S328 } \\
\text { (VLA) }\end{array}$ & $\begin{array}{c}\text { S1287 } \\
\text { (GMRT) }\end{array}$ & $\alpha_{328}^{74}$ & $\alpha_{1287}^{328}$ & $\mathrm{t}_{R E} / \mathrm{t}_{C I}$ \\
\hline $0128 \mathrm{~A}$ & 012834.551 & -253822.90 & $1200(190)$ & $129(9)$ & $5.5(0.45)$ & $-1.5(.15)$ & $-2.3(.15)$ & 1.9 \\
$0128 \mathrm{~B}$ & 012824.697 & -253911.15 & $650(160)$ & $152(7.5)$ & $31.0(0.3)$ & $-1.0(.2)$ & $-1.2(.05)$ & 0.5 \\
$0128 \mathrm{C}$ & 012831.112 & -254031.80 & $180(75)$ & $35.8(2.1)$ & $4.4(0.4)$ & $-1.1(.34)$ & $-1.5(.15)$ & 1.6 \\
0439 & 043953.930 & 530412.02 & $1600(180)$ & $95.6(4.6)$ & $7.8(0.75)$ & $-1.9(.1)$ & $-1.8(.14)$ & 0.8 \\
1114 & 111413.194 & 151944.12 & $600(101)$ & $43.3(6)$ & $2.1(0.46)$ & $-1.8(.2)$ & $-2.2(.27)$ & 0.4 \\
1133 & 113345.045 & 232514.05 & $1400(118)$ & $150.8(12)$ & $18.1(0.40)$ & $-1.5(.1)$ & $-1.6(.09)$ & 1.6 \\
1152 & 115236.104 & 373246.63 & $4300(102)$ & $387(11)$ & $25.5(2.4)$ & $-1.6(.03)$ & $-1.9(.08)$ & 2.5 \\
2216 & 221658.007 & -172508.03 & $10800(380)$ & $420(30)$ & $15.9(0.7)$ & $-2.2(.07)$ & $-2.4(.09)$ & 0.2 \\
2313 & 231346.837 & 384216.13 & $1800(100)$ & $245(12)$ & $28.7(8.5)$ & $-1.3(.08)$ & $-1.6(.25)$ & 1.5 \\
2345 & 234515.392 & 215739.82 & $2900(114)$ & $257(20)$ & $9.6(1.6)$ & $-1.6(.08)$ & $-2.4(.18)$ & 1.8 \\
\hline
\end{tabular}

Note. - All flux densities are integrated values and in mJy. Values within parentheses are $1 \sigma$ error estimates. The sources with optical identifications are 0128B (2dFGRS S149Z127, $\mathrm{z}=0.207$ ), 1152 (SDSS $\mathrm{J} 115236.45+373243.7, \mathrm{z}=0.229$ ), 2216 (RBS 1842, $\mathrm{z}=0.136$ ), and 2345 (2MASX J23451645+2157578, $\mathrm{z} \sim 0.15$ (based on the k-z relation of van Breugel et al (1999))). 